\title{
Fibrosis muscular yatrogénica: aproximación experimental
}

Dr. Luis Miguel Cartier*; Ricardo Zalaquet, ${ }^{* *}$ Ricardo Sepúlveda, ${ }^{* * *}$ Carmen Vergara.

\begin{abstract}
An histopathological study of three weeks old rat's striate muscle subject to the injection with penicillin and chloramphenicol both in heparinized and non-heparinized animals is presented.

These antibiotics are capable of inducing muscle necrosis and an intersticial reaction which is enhanced by the presence of hemorrhage.

The suggestion is made that intrumuscular antibiotics both in the new born and infiant are able to induce iatrogenic fibrosis ot the cuadriceps muscle.
\end{abstract}

La fibrosis muscular yatrogénica es un cuadro clínico que se presenta en niños que pueden tener el antecedente en la etapa de reciér nacido o lactante menor, de tratamientos inyectables por vía intramuscular, habitualmente de antibióticos.

Trabajo realizado en el Departangento de Medicina Experimental y en el Laboratorio de Neuropatologia del Departamento de Ciencias Neurológicas de la Facultad de Medicina, Sede Oriente de la Universidad de Chile.

*Servicio de Neurología, Hospital del Salvador. Facultad de Medicina Oriente. Universidad de Chile.

**Interno Facultar de Medicina Oriente. Universidad de Chile. ***Universidad de Chile. Sede Antofagasta.

****Tecnólogo Médica. Servicio de Neurología. Hospital del Salvador.
Se considera por la mayoría de los autores que uno de los factores etiopatogénicos de esta enfermedad estaría en relación a la intensidad de estos tratamientos. 1, 2, 3, 4, 5, $6 \mathrm{Si}$ bien el cuadro tiene su máxima expresión clínica en relación al músculo cuadriceps, expresado en una pérdida progresiva de la flexión de la rodilla, 2, 3, también se ha observado en relación a la musculatura glútea. ${ }^{3} \mathrm{Se}$ acepta que los recién nacidos prematuros $y$ de bajo peso son los que estarían más expuestos a desarrollar estas lesiones. ${ }^{4,5}$

El estudio histológico ha revelado como hallazgos más destacados el desarrollo de una fibrosis, infiltración grasa y atrofia de las fibras musculares. 2, 3, 4,5 
Por otra parte, en autopsias practicadas a recién nacidos o lactantes que han fallecido como corrsecuencia de infecciones graves y que habían sido tratados en forma intensiva y prolongada $\mathrm{com}$ antibióticos intramusculares, se ha observado la presencia de hematoma en los músculos puncionados.' Se ha invocado como un factor etiopatogénico la organización del hematoma, también la compresión vascular y la isquemia subsecuente, deterninada por este, analogándolo a la enfermedad de Volkmann. ${ }^{4}$ ' Se sabe que las manifestaciones de tipo hemorragíparo son fácilmente provocadas en este periodo de la vickt, principalmente en los prematuros, las que podrian ser intensifjcadas por un proceso infeccioso.

A modo de aproximación experimental a esta patología un grupo de ratas de 3 semanas de vida fiue sometido a inyecciones intramusculares en la región posterior interna del muslo con el objeto de observar las alteraciones producidas a corto, mediano y largo plazo. Se utilizó para esto la penicilina sódica y el succionto de cloramfenicol, por ser los antibióticos de uso más difindido en la práctica clínica y solución de $\mathbf{X a C l} 9 \%$ como elemento de comparación, con objeto de establecersi la naturaleza química de la sustancia inyectada juega algún rol en el desarrollo de esta lesión, que ha sido descartada por ciertos autores. ${ }^{4}$

En forma simultánea, otro grupo de ratas de iguales características, fue sometido a un procedimiento idéntico, pero éstas además fueron he- parinizadas con el objeto de establecer si efectivamente una condición hemorragípara es un factor determinante en el desarrollo de esta patología.

\section{MATERLAL Y METODO}

Se tomaron 30 ratas de 3 semanas de vida, sin consideración de sexo. Se dividieron en dos gripos iguales. Uno se trató con heparina intraperitoneal en dosís única diaria de $1 \mathrm{mg}$ durante 10 días. Cada grupo, se dividió en 3 subgrupos de 5 ratas, en los que se procedió de la siguiente manera:

$$
\begin{aligned}
& \text { - Subgrupo 1: Penicilina sódica } \\
& 100.000 \mathrm{Ul} / 12 \mathrm{~h} \times 10 \text { días } \\
& \text { - Subgrupo 2: Cloramfenicol succinato } 4,5 \mathrm{mg} / 12 \mathrm{~h} \times 10 \text { dias } \\
& \text { - Subgrupo 3: } \text { Solución } \mathrm{NaCl} 9 \% \\
& 0,5 \mathrm{ml} / 12 \mathrm{~h} \times 10 \text { días }
\end{aligned}
$$

Tanto la dosis de heparina como la de los antibióticos se calcularon en forma aproximada a partir de la siguiente ecuación:

Dosis $=a \cdot w^{0,734(9)}$

En que "a" es la dosis utilizada habitualmente en infecciones graves en recién nacidos, $y$ " $w$ " es el peso del animal (rata). Para esto último se consideró un peso promedio de $40 \mathrm{~g}$. Las dosis resultantes se ajustaron a un volumen de $0,5 \mathrm{ml}$. La figura $N{ }^{\circ}$ l resume el material biológico y metódica utilizados.

\section{Figura 1}

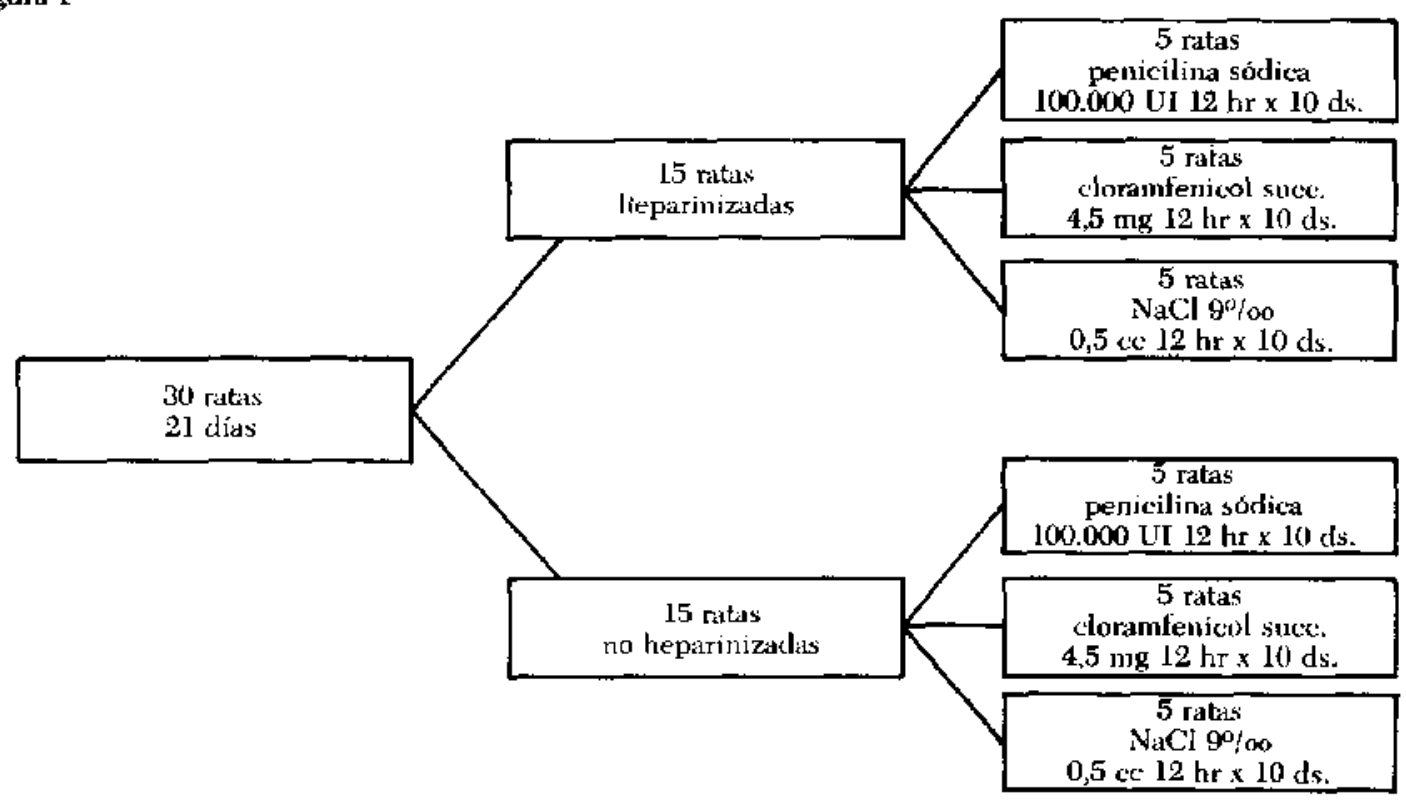


El sitio de punción escogido fue la musculatura de la región posterointerna del muslo iz quierdo de la rata ${ }^{10}$ y no la región anteroextema, por razones técnicas. Como instrumental de punción se utilizaron agujas intradérmicas finas.

Durante todo el desarrollo del procedimiento se estuvo atento a la aparición de trastomos en la marcha, formación de bematoma o cualquier otra alteración de importancia. Las ratas fueron sacrificadas al día siguiente, al mes y a los 5 meses de finalizada la fase experimental. En cada oportunidad se efectuó un examen macroscópico y microscópico de los músculos utilizados. La musculatura homóloga de la extremidad inferior derecha se tomó como patrón de comparación. Para el estudio histológico se fijó la musculatura posterior en bloque en solución de Buoin, se incluyó en parafina y se hicieron cortes seriados de la muestra, los que se tiñeron con H.E., Gomori y V.G.

\section{RESULTADOS}

Al cuarto día de experimentación 6 de las ratas heparinizadas presentaron hematoma del muslo izquierdo, 4 de las cuales estaban recibiendo penicilina sódica y dos soluciones de $\mathrm{NaCl} 9 \%$, que se resolvieron rápida y espontáneamente. No se observó alteración de la marcha ni de la motilidad de la extremidad, excepto en los momentos inmediatamente siguientes a la inyección.

El examen macroscópico de los músculos puncionados fue positivo en todas las ratas, independientemente de la sustancia inyectada. Los hallazgos consistieron en ligera equímosis de las envolturas aponeuróticas y congestión de los músculos, los que fueron algo mayor en las ratas heparinizadas. No se observaron lesiones ni cambios significativos en los músculos de las ratas sacrificadas al mes y a los 5 meses.

Para el estudio histológico se consideraron los siguientes parámetros:

1) Compromiso de la fibra muscular: a) necrosis, b) reacción inflamatoria, c) atrofia

2) Compromiso estructural de vasos y nervios

3) Presencia de hemorragia o hemosiderina

4) Reacción del tejido conjuntivo instersticial.

A. Grupo de ratas sacrificadas al día siguiente de finalizada la fase experimental, no heparinizadas, tenían las siguientes manifestaciones según la sustancia inyectada: a) Penicilina: zonas de fibras musculares necrosadas asociadas a infiltrados intersticiales, y en algunos sectores fibras de regeneración con clara atrotia de las fibras del sector. No hay signos que sugieran hemorragia. Se aprecia la proliferación d'el tejido conjuntivo intersticial traducida en engrosamiento de las vainas interfasciculares. (Fig. 2.)

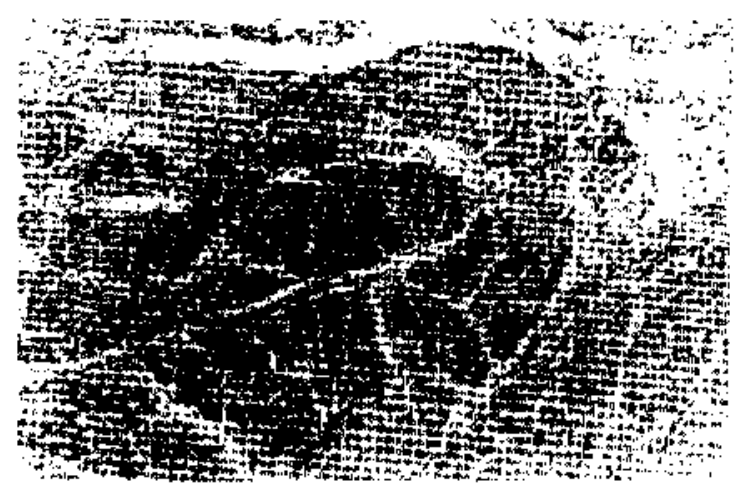

Figura 2. Músculo de rata inyectada con pentcilina. Sacrificuda a los 10 días. H.E.X.A.

b) Cloramfenicol: zonas de necrosis localizadas asociada a infiltrado linfocitario y a atrofia de las fibras musculares. No se comprueba alteración vascular ni existencia de hemosiderina. Hay reacción intersticial del tejido conjuntivo limitada a los sectores lesionados. (Fig. 3.)

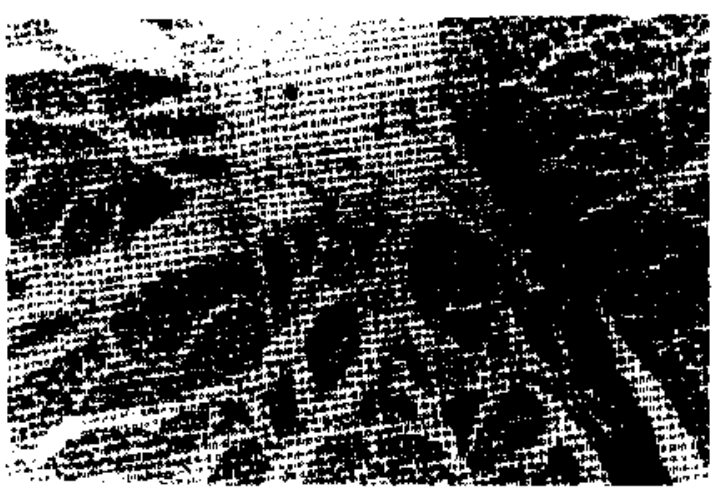

Figura 3. Rata inyectada con cloranfenical. Sacrificada a los 10 días. H.E.X.4.

c) NaCl 9\%: no se observa lesión muscular. En algunos vasos hay infiltrado perivascular, $y$ depósitos de hemosiderina en un ganglio satélite de la muestra.

B. Ratas heparinizadias cuyos hallazgos histológicos consistieron en:

a) Penicilina: sectores de fibras musculares necróticas, con infiltrado linfocitario poco impor- 
tante. Se aprecia atrofia de fibras musculares y algunas fibras regenerativas. Los paquetes vasculonervioso muestran reacción instersticial y hay depósitos de hemosiderina. El engrosamiento de vainas y proliferación del tejido combuntivo son marcados (Fig. 4.)

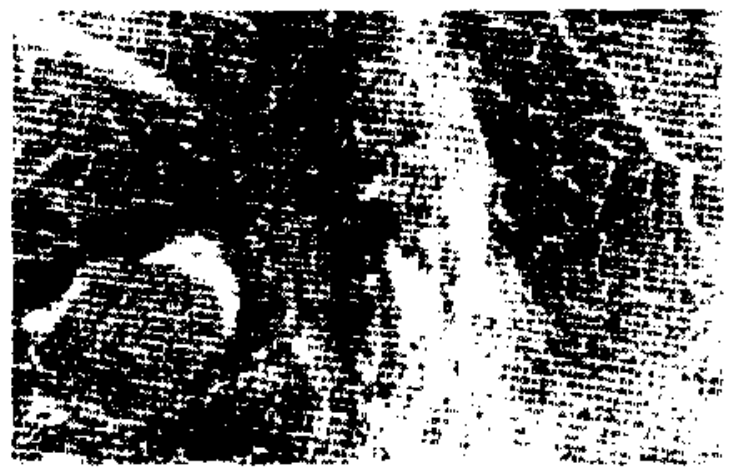

Figura 4. Músculo de rata inyectada con heparina y penicilina. Sacrificada a las 10 días. H.E.X.10

b) Cloramfenicol: se observa un fascículo muscular con necrosis y atrofia e infiltración, pero lo más destacado es el engrosaniento importante del tejido intersticial asociado a la presencia de abundantes depósitos de hemosiderina. En algunos sectores el engrosamiento de las vai- nas se transforma en verdaderas bandas fibrosas (Fig. 5.)

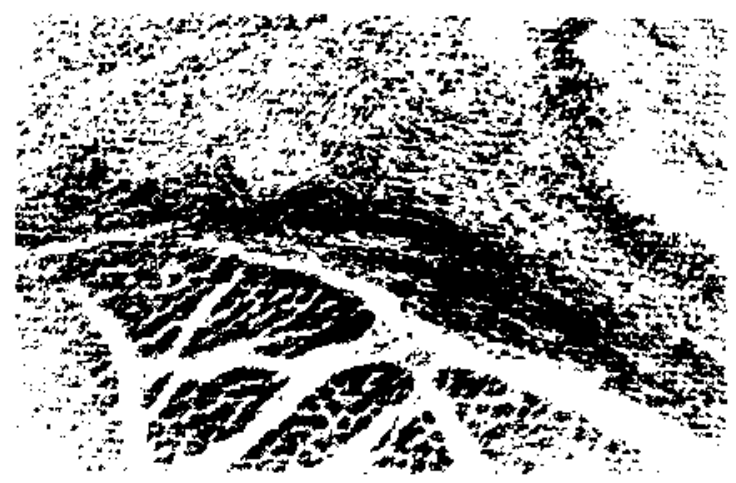

Figura 5. Mitisculo de rata inyectada con heparina y cluramfeni(o)]. Sacritiecadla a los 10 dias. H.E.X.4.

c) $\mathrm{NaCl} 9 \%$ : no se comprueba compromiso del tejido muscular. Sólo se aprecia infiltrado instersticial y perivascular de mediana intensidad, y proliferación del tejido conjuntivo en relación a la presencia de hemosiderina, la que es acentuada, especialmente en los pedículos vasculonervioso.

La Tabla $N .{ }^{\circ}$ l es un resumen comparativo de los hallazgos histológicos encontrados en estas ratas.

Tabla 1

Ratas sucrificadas al día siguiente de finalizado el procedìmiento experimental.

\begin{tabular}{|c|c|c|c|c|c|c|}
\hline ratas & $\begin{array}{l}\text { necrosis } \\
\text { muscular }\end{array}$ & $\begin{array}{l}\text { infiltrado } \\
\text { lintocitario }\end{array}$ & $\begin{array}{l}\text { atrofia } \\
\text { minscular }\end{array}$ & $\begin{array}{l}\text { reaccion de } \\
\text { pague te } \\
\text { wasc-riavioso }\end{array}$ & $\begin{array}{l}\text { hernormgia } \\
\text { hernosiderino }\end{array}$ & $\begin{array}{l}\text { engrosamien- } \\
\text { to koinas tej } \\
\text { instertirty }\end{array}$ \\
\hline peniciling & $t$ & + & $t$ & & & + \\
\hline cloramtenical & + & + & + & & & $t$ \\
\hline Nis C! $9 \%$ & & + & & & + & \\
\hline $\begin{array}{l}\text { heparina } \\
\text { penicilina }\end{array}$ & + & + & + & + & $t$ & + \\
\hline $\begin{array}{l}\text { heparina } \\
\text { cloramenten }\end{array}$ & + & + & + & + & + & + \\
\hline $\begin{array}{l}\text { heparina } \\
\mathrm{NaCl} \quad 9 \%\end{array}$ & & $t$ & & + & $t$ & + \\
\hline
\end{tabular}

C. En el grupo de ratas que fueron sacriticadas al mes (Tabla 2) los hallazgos consistieron fun- damentalmente en atrofia muscular en aquellas que habían sido inyectadas con penicilina 
(Fig. 6), Cloramfenicol y heparina-Cloramfenicol (Fig. 7), y el engrosamiento de vainas y reacción intersticial en las que recibieron cua-

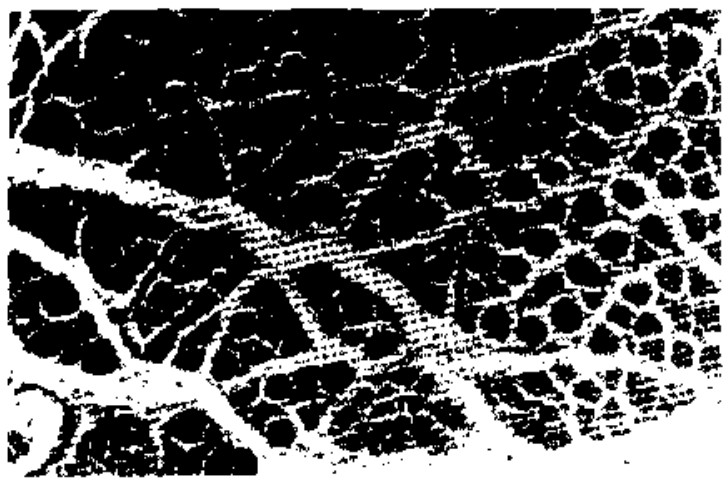

Figura 6. Músculo de rata inyectada con penicilina. Sacrificada al mes. H.E.X. 10. lesquiera de los dos antibióticos, hepariniziadas 0 no. No hubo otras alteraciones signiticativas en este grupo de ratas.

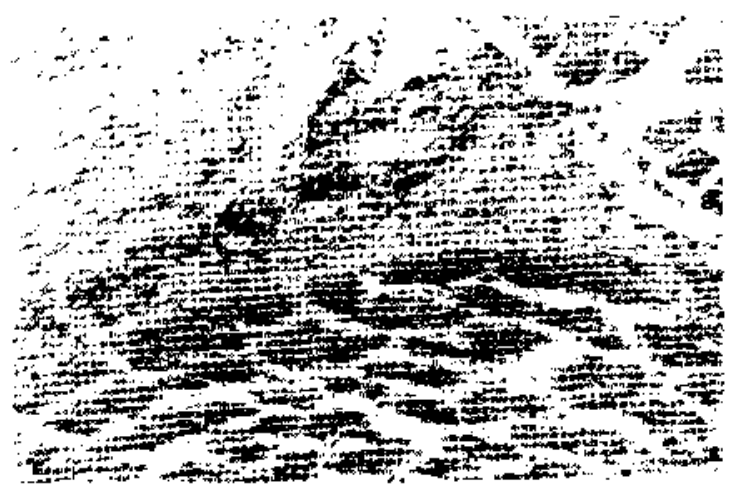

Figura 7. Mrisculo de rata inyecteda con cloranatenicyl. Sacriticadit al mes. H.E.X.10.

Tabla 2

Ratas sacríficadas al mes de finalizado el procedimiento experimental.

\begin{tabular}{|c|c|c|c|c|c|c|}
\hline ratas & $\begin{array}{l}\text { riecrosis } \\
\text { muscular }\end{array}$ & $\begin{array}{l}\text { intiltrado } \\
\text { in tocitario }\end{array}$ & $\begin{array}{c}\text { atrofia } \\
\text { muscular }\end{array}$ & $\begin{array}{l}\text { reacciol de } \\
\text { paquete } \\
\text { vasc-nervioso }\end{array}$ & $\begin{array}{l}\text { hemor ragia } \\
\text { hemosiderino }\end{array}$ & $\begin{array}{l}\text { engrosamjen } \\
\text { to vai mas tej } \\
\text { instersti cial }\end{array}$ \\
\hline penicilina & & & & & & \\
\hline clorantenico & & & & & & \\
\hline $\mathrm{Na}$ Cl $9 \%$ & & & & & & \\
\hline $\begin{array}{l}\text { he parina } \\
\text { penicilina }\end{array}$ & & & & & & $t$ \\
\hline $\begin{array}{l}\text { heparina } \\
\text { cloranfenica }\end{array}$ & & & & & & + \\
\hline $\begin{array}{c}\text { heparina } \\
9: \%\end{array}$ & & & & & & \\
\hline
\end{tabular}

D. En las ratas sacrificadas a los 5 meses no se observó una variación de importancia respecto de las alteraciones encontradas en aquellas que lo fueron al mes.

Se observó, en forma categórica, que la intensidad de las lesiones eran menores mientras más alejadas del procedimiento experimental eran sacrificadas las ratas para ser sometidas a estudios.

\section{CONCLUSIONES Y COMENTARIO}

Se concluye que tanto la penicilina sódica como el succinato de cloranfenicol producen una acción claramente deletérea sobre las fibras musculares estriadas, y una reacción intersticial secundaria, en ratas de alrededor de 3 semanas de vida, que resultó de mayor intensidad en aquellas que fueron simultáneamente heparinizadas. Sin embargo, se aprecia que se produce una buena reparación del tejido muscular en el transcurso del tiempo. Por otra parte, se puede inferir que un tratamiento más intenso y prolongado puede producir daños irreparables en la estructura muscular y en los payuetes vasculonervioso adyacentes.

Pensamos, de acuerdo a los antecedentes bibliográficos, que la situación en los niños someti- 
dos a tratamiento intensivo de antibióticos intramusculares es de mayor riesgo que la condición experimental expuesta. Ello hace presumir que las dosis de antibióticos utilizadas producen necrosis muscular y que una condición hemorragipara de base acentúa claramente la reacción intersticial, al parecer por la sula presencia de hemosiderina en estos tejidos. Asimismo, que esta sitn:ación debe estar intensificada en los recién nacidos $y$ lactantes menores con cuadros toxiinfecciosos y a veces desnutrición.

La reparación que se realiza de manera adecuada en las ratas normales y bien alimentadas. seguramente no se expresa de la misma manera en estos niños. Creemos que los factores vascular y de inervación del músculo juegan algún papel. Tambien es presumible que las condiciones individuales predisponentes y desencadenadas por la agresión terapéutica sean otro factor en la producción de esta fibrosis nusculares.

\section{RESUMEN}

Se hace estudio histopatológico de músculo estriado en ratas de 3 semanas de vida, heparinjzadas y sin heparina, inyectadas por vía intramuscular con penicilina, cloramfenicul y $\mathrm{NaCl} 9 \%$.

Se concluye que estos antibióticos son capaces de producir necrosis muscular y una reacción intersticial secundaria, que es acentuada por la presencia de hemorragia.

Se intenta extrapolar esta situación experimental a lo que sucede con tratamiento antibiótico intramuscular intensivo y prolongado en el recién nacido y lactante menor, que a nuestro juicio y el de los tratadistas sobre el tema sería una de la causa de la tibrosis yatrogénica del cuádriceps.

\section{REFERENCIAS}

1 Gimn, D. R. Contrachire of the quadticeps murclk. J Bune Jt. sury. $46 \mathrm{~B}, 492-497,1964$.

2 Lisyd-Rolerts, G. C. y Thomas, T.G. The aetiology of cu:idriceps contrachuse in childrer. J. Bone Jt. Surg. 46 B, 498502.1964.

3 Theoderm, S. D. Fibrose et retraction du cijadricreps consectitives a des injections intramuscinlares chez les nourtisons. Helv. Paediat. Acta 24: 3(6-310, 1969.

4 Sec, $G, \varepsilon t$ al. Fibrose du yualriceps consécutive a des injections intramusculaires practiqués chez le prematuré et le neurtison. Sem. Zlosp. Paris, Ann. Pédiat. 44: 104-110, 1968\%.

5 Sasnders, F. P., Hoffnagel et Staples, O. S. Progressive tibrasis of the cpuadriceps temuris. J. Bone Jt. Surg. $47 \mathrm{~A}, 380-384$, 1965.

6 Agtrilera, C. y Sepritata, R. Fibrosis Yatrogentica del Cuádriceps. Rev. Chil. Ped. Vol. 46, N.* 4: 357-362, 1975.

- INeséshelo.J. Compunicación personal.

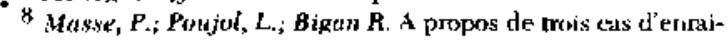
dissement en extension du genou par tibroste pmogessive du Gitadriceps. Arch, franc. Pédiat, 1965, 22, 697-705.

Guather, B. On theories of Biological Similarities. 1. Ed. Gearg Ghieme: Leizig. 1975.

10 Farris, H., Griffith,J. The Ratin the Laboratory Investigation. 2. Ed. J. B. Isippincott Campany, Philaxdelphia, Pág. 28 y 49. 